\title{
Quark-Lepton Nonuniversality
}

\author{
Xiao-Yuan Li \\ Institute of Theoretical Physics, Chinese Academy of Sciences, Beijing, China \\ and Ernest $\mathrm{Ma}$ \\ Physics Department, University of California, Riverside, California 92521, USA
}

Received on 6 December, 2003.

\begin{abstract}
There is new experimental evidence which may be interpreted as a small departure from quark-lepton universality. We propose to understand this as the result of a hierarchy of mass scales in analogy to $m_{u}, m_{d}<<\Lambda_{Q C D}$ for strong isospin. We show $\left(G_{F}\right)_{l q}^{N C}<\left(G_{F}\right)_{l q}^{C C}<\left(G_{F}\right)_{l l}^{C C}<\left(G_{F}\right)_{l l}^{N C}$ in principle, but all are still approximately equal. New physics is predicted at the $\mathrm{TeV}$ scale.
\end{abstract}

\section{Introduction}

In the Standard Model, the low-energy effective weak interactions are of the form

$$
\mathcal{H}_{\text {int }}=\frac{4 G_{F}}{\sqrt{2}}\left[j^{(+)} j^{(-)}+\left(j^{(3)}-\sin ^{2} \theta_{W} j^{(e m)}\right)^{2}\right],
$$

where

$$
\frac{4 G_{F}}{\sqrt{2}}=\frac{g^{2}}{2 M_{W}^{2}}=\frac{g^{2}+g^{\prime 2}}{2 M_{Z}^{2}}=\frac{1}{v^{2}} .
$$

Note that $G_{F}$ is independent of $g$ and $g^{\prime}$.

As a result of Eq. (1), there are 3 predictions:

$$
\begin{aligned}
& \text { (A) } G_{F}^{q}=G_{F}^{l}, \quad \sin ^{2} \theta_{W}^{q}=\sin ^{2} \theta_{W}^{l} \\
& \text { (B) } G_{F}^{e}=G_{F}^{\mu}=G_{F}^{\tau} \\
& \text { (C) } G_{F}^{C C}=G_{F}^{N C}
\end{aligned}
$$

Possible experimental deviations of $(\mathrm{A})$ and $(\mathrm{C})$ have now been observed at the $3 \sigma$ level. Whereas it is too early to tell for sure that these are real effects, it is clearly desirable to have a theoretical framework where departures from quarklepton universality are naturally expected and which reduces to the Standard Model in the appropriate limit.

\section{Three Experimental Discrepancies}

(1) A recent measurement [1] of the neutron $\beta$-decay asymmetry has determined that

$$
\left|V_{u d}\right|=0.9713(13),
$$

which, together with [2] $\left|V_{u s}\right|=0.2196(23)$ and $\left|V_{u b}\right|=$ $0.0036(9)$, implies the apparent nonunitarity of the quark mixing matrix, i.e.

$$
\left|V_{u d}\right|^{2}+\left|V_{u s}\right|^{2}+\left|V_{u b}\right|^{2}=0.9917(28) .
$$

However, if $\left(G_{F}\right)_{l q}^{C C}<\left(G_{F}\right)_{l l}^{C C}$, as we will show, then the above is actually expected.

(2) The NuTeV experiment [3] which measures $\nu_{\mu}$ and $\bar{\nu}_{\mu}$ scattering on nucleons reported a value of

$$
\sin ^{2} \theta_{W}=0.2277 \pm 0.0013 \pm 0.0009,
$$

as compared to the Standard-Model expectation of $0.2227 \pm$ 0.00037 , assuming that $\left(G_{F}\right)_{l q}^{N C} /\left(G_{F}\right)_{l q}^{C C}=1$. In our model, this ratio will be smaller than one, which would explain the data if it is $0.9942 \pm 0.0013 \pm 0.0016$ and $\sin ^{2} \theta_{W}$ does not change. However, we do expect the latter to change, but since its precise determination comes from $Z$ decay, we need to consider also data at the $Z$ resonance.

(3) In precision measurements of $e^{-} e^{+} \rightarrow Z \rightarrow q \bar{q}$ and $l \bar{l}$, there seem to be two different values of $\sin ^{2} \theta_{\text {eff }}$, i.e. [4]

$$
\begin{aligned}
\left(\sin ^{2} \theta_{\text {eff }}\right)_{\text {hadrons }} & =0.23217(29) \\
\left(\sin ^{2} \theta_{\text {eff }}\right)_{\text {leptons }} & =0.23113(21) .
\end{aligned}
$$

This may be an indication of a small deviation from quarklepton universality.

In this talk I will show that (1) is naturally explained by a gauge model of quark-lepton nonuniversality [5], the prototype of which was proposed over 20 years ago [6] for generation nonuniversality. As a result, effects indicated by (2) and (3) are also expected, but the observed deviations are too large.

\section{Gauge Model of Quark-Lepton Nonuniversality}

Consider the gauge group $S U(3)_{C} \times S U(2)_{q} \times S U(2)_{l} \times$ $U(1)_{q} \times U(1)_{l}$ with couplings $g_{s}$ and $g_{1,2,3,4}$ respectively. 
The quarks and leptons transform as

$$
\begin{aligned}
(u, d)_{L} & \sim(3,2,1,1 / 6,0), \\
u_{R} & \sim(3,1,1,2 / 3,0), \\
d_{R} & \sim(3,1,1,-1 / 3,0), \\
(\nu, e)_{L} & \sim(1,1,2,0,-1 / 2), \\
e_{R} & \sim(1,1,1,0,-1) .
\end{aligned}
$$

The scalar sector consists of

$$
\begin{aligned}
\left(\phi_{1}^{+}, \phi_{1}^{0}\right) & \sim(1,2,1,1 / 2,0), \\
\left(\phi_{2}^{+}, \phi_{2}^{0}\right) & \sim(1,1,2,0,1 / 2), \\
\chi^{0} & \sim(1,1,1,1 / 2,-1 / 2),
\end{aligned}
$$

and a bidoublet

$$
\eta=\frac{1}{\sqrt{2}}\left(\begin{array}{cc}
\eta^{0} & -\eta^{+} \\
\eta^{-} & \bar{\eta}^{0}
\end{array}\right) \sim(1,2,2,0,0),
$$

which is assumed to be self-dual, i.e. $\eta=\tau_{2} \eta^{*} \tau_{2}$. Note that $g_{1}$ may be different from $g_{2}$, and $g_{3}$ may be different from $g_{4}$, so there is no quark-lepton symmetry at this level. The remarkable fact is that the effective low-energy weak interactions of the quarks and leptons will turn out to be independent of $g_{1,2,3,4}$ and become all equal in a certain limit, as shown below.

Consider

$$
\left\langle\phi_{1,2}^{0}\right\rangle=v_{1,2},\left\langle\chi^{0}\right\rangle=w,\left\langle\eta^{0}\right\rangle=u,
$$

then the $2 \times 2$ charged-gauge-boson mass-squared matrix is given by

$$
\mathcal{M}_{W}^{2}=\frac{1}{2}\left[\begin{array}{cc}
g_{1}^{2}\left(v_{1}^{2}+u^{2}\right) & -g_{1} g_{2} u^{2} \\
-g_{1} g_{2} u^{2} & g_{2}^{2}\left(v_{2}^{2}+u^{2}\right)
\end{array}\right] .
$$

Thus the effective lepton-lepton charged-current weakinteraction strength, i.e. that of $\mu$ decay, is

$$
\frac{4\left(G_{F}\right)_{l l}^{C C}}{\sqrt{2}}=\frac{g_{2}^{2}}{2}\left(\mathcal{M}_{W}^{-2}\right)_{22}=\frac{u^{2}+v_{1}^{2}}{\left(v_{1}^{2}+v_{2}^{2}\right) u^{2}+v_{1}^{2} v_{2}^{2}},
$$

whereas the analagous expression for nuclear $\beta$ decay is

$\frac{4\left(G_{F}\right)_{l q}^{C C}}{\sqrt{2}}=\frac{g_{1} g_{2}}{2}\left(\mathcal{M}_{W}^{-2}\right)_{12}=\frac{u^{2}}{\left(v_{1}^{2}+v_{2}^{2}\right) u^{2}+v_{1}^{2} v_{2}^{2}}$.

Note that both are independent of $g_{1}$ and $g_{2}$, and their ratio is not one, but rather

$$
\frac{\left(G_{F}\right)_{l q}^{C C}}{\left(G_{F}\right)_{l l}^{C C}}=\frac{u^{2}}{u^{2}+v_{1}^{2}} \simeq 1-\frac{v_{1}^{2}}{u^{2}} .
$$

The apparent nonunitarity of the quark mixing matrix, i.e. Eq. (7), is then naturally explained with

$$
\frac{v_{1}^{2}}{u^{2}}=0.0042(14)
$$

As for the effective neutral-current interactions, we have

$$
\begin{aligned}
& \frac{4\left(G_{F}\right)_{l q}^{N C}}{\sqrt{2}}=\frac{u^{2} w^{2}}{\left(v_{1}^{2}+v_{2}^{2}\right) u^{2} w^{2}+v_{1}^{2} v_{2}^{2}\left(u^{2}+w^{2}\right)} \\
& \simeq \frac{4\left(G_{F}\right)_{\mu}}{\sqrt{2}}\left[1-\frac{v_{1}^{2}}{u^{2}}-\left(\frac{v_{2}^{2}}{v_{1}^{2}+v_{2}^{2}}\right) \frac{v_{1}^{2}}{w^{2}}\right], \\
& \frac{4\left(G_{F}\right)_{l l}^{N C}}{\sqrt{2}}=\frac{u^{2} w^{2}+v_{1}^{2}\left(u^{2}+w^{2}\right)}{\left(v_{1}^{2}+v_{2}^{2}\right) u^{2} w^{2}+v_{1}^{2} v_{2}^{2}\left(u^{2}+w^{2}\right)} \\
& \simeq \frac{4\left(G_{F}\right)_{\mu}}{\sqrt{2}}\left[1+\left(\frac{v_{1}^{2}}{v_{1}^{2}+v_{2}^{2}}\right) \frac{v_{1}^{2}}{w^{2}}\right] .
\end{aligned}
$$

This implies that the ratio

$$
\frac{\left(G_{F}\right)_{l q}^{N C}}{\left(G_{F}\right)_{l q}^{C C}} \simeq 1-\left(\frac{v_{2}^{2}}{v_{1}^{2}+v_{2}^{2}}\right) \frac{v_{1}^{2}}{w^{2}}
$$

is what $\mathrm{NuTeV}$ actually measures [3]. The corresponding $\sin ^{2} \theta_{W}$ expressions depend on the identification of the observed $Z$ boson as a linear combination of the 3 massive neutral gauge bosons of this model, which will be discussed in the next section.

\section{Observables at the $Z$ Pole}

There are 4 electroweak gauge couplings in this model. The electromagnetic coupling $e$ is given by

$$
\frac{1}{e^{2}}=\frac{1}{g_{1}^{2}}+\frac{1}{g_{2}^{2}}+\frac{1}{g_{3}^{2}}+\frac{1}{g_{4}^{2}}
$$

Defining $g_{i j}^{-2} \equiv g_{i}^{-2}+g_{j}^{-2}$, the photon $A$ and 3 orthonormal $Z$ bosons are given in the basis $\left(W_{q}^{0}, W_{l}^{0}, B_{q}, B_{l}\right)$ by

$$
\begin{aligned}
A & =e\left(\frac{1}{g_{1}}, \frac{1}{g_{2}}, \frac{1}{g_{3}}, \frac{1}{g_{4}}\right), \\
Z_{1} & =e\left(\frac{g_{12}}{g_{34} g_{1}}, \frac{g_{12}}{g_{34} g_{2}}, \frac{-g_{34}}{g_{12} g_{3}}, \frac{-g_{34}}{g_{12} g_{4}}\right), \\
Z_{2} & =g_{12}\left(\frac{1}{g_{2}}, \frac{-1}{g_{1}}, 0,0\right), \\
Z_{3} & =g_{34}\left(0,0, \frac{1}{g_{4}}, \frac{-1}{g_{3}}\right) .
\end{aligned}
$$

The observed $Z$ boson is approximately $Z_{1}-\epsilon_{2} Z_{2}-\epsilon_{3} Z_{3}$, where

$$
\begin{aligned}
& \epsilon_{2} \simeq \frac{g_{34} g_{12}^{4}}{e g_{1}^{3} g_{2}^{3}}\left(\frac{g_{1}^{2} v_{1}^{2}-g_{2}^{2} v_{2}^{2}}{u^{2}}\right), \\
& \epsilon_{3} \simeq \frac{g_{12} g_{34}^{4}}{e g_{3}^{3} g_{4}^{3}}\left(\frac{-g_{3}^{3} v_{1}^{2}+g_{4}^{2} v_{2}^{2}}{w^{2}}\right) .
\end{aligned}
$$

Deviations from the Standard Model must occur and quarklepton universality in $Z$ decay is violated if $\epsilon_{2} \neq 0$ or $\epsilon_{3} \neq 0$.

We have obtained [5] all the appropriate expressions for the expected deviations from the Standard Model in terms of 5 parameters:

$$
\frac{v_{1}^{2}}{u^{2}}, \frac{v_{1}^{2}}{w^{2}}, r \equiv \frac{v_{2}^{2}}{v_{1}^{2}}, y \equiv \frac{g_{2}^{2}}{g_{1}^{2}+g_{2}^{2}}, x \equiv \frac{g_{4}^{2}}{g_{3}^{2}+g_{4}^{2}},
$$


Table I. Fit Values of 22 Observables

\begin{tabular}{cccccc}
\hline Observable & Measurement & Standard Model & Pull & This Model & Pull \\
\hline$\Gamma_{l}[\mathrm{MeV}]$ & $83.985 \pm 0.086$ & 84.015 & -0.3 & 83.950 & +0.4 \\
$\Gamma_{\text {inv }}[\mathrm{MeV}]$ & $499.0 \pm 1.5$ & 501.6 & -1.7 & 501.2 & -1.5 \\
$\Gamma_{h a d}[\mathrm{GeV}]$ & $1.7444 \pm 0.0020$ & 1.7425 & +1.0 & 1.7444 & -0.0 \\
$A_{f b}^{0, l}$ & $0.01714 \pm 0.00095$ & 0.01649 & +0.7 & 0.01648 & +0.7 \\
$A_{l}\left(P_{\tau}\right)$ & $0.1465 \pm 0.0032$ & 0.1483 & -0.6 & 0.1482 & -0.5 \\
$R_{b}$ & $0.21644 \pm 0.00065$ & 0.21578 & +1.0 & 0.21582 & +1.0 \\
$R_{c}$ & $0.1718 \pm 0.0031$ & 0.1723 & -0.2 & 0.1722 & -0.1 \\
$A_{f b}^{0, b}$ & $0.0995 \pm 0.0017$ & 0.1040 & -2.6 & 0.1039 & -2.6 \\
$A_{f b}^{0, c}$ & $0.0713 \pm 0.0036$ & 0.0743 & -0.8 & 0.0740 & -0.8 \\
$A_{b}$ & $0.922 \pm 0.020$ & 0.935 & -0.7 & 0.934 & -0.6 \\
$A_{c}$ & $0.670 \pm 0.026$ & 0.668 & +0.1 & 0.665 & +0.2 \\
$A_{l}(\mathrm{SLD})$ & $0.1513 \pm 0.0021$ & 0.1483 & +1.4 & 0.1482 & +1.5 \\
$\sin ^{2} \theta_{\text {eff }}^{\text {lept }}\left(Q_{f b}\right)$ & $0.2324 \pm 0.0012$ & 0.2314 & +0.8 & 0.2322 & +0.2 \\
$m_{W}[\mathrm{GeV}]$ & $80.449 \pm 0.034$ & 80.394 & +1.6 & 80.390 & +1.7 \\
$\Gamma_{W}[\mathrm{GeV}]$ & $2.139 \pm 0.069$ & 2.093 & +0.7 & 2.093 & +0.7 \\
$g_{V e}^{\nu e}$ & $-0.040 \pm 0.015$ & -0.040 & -0.0 & -0.039 & -0.1 \\
$g_{A}^{\nu e}$ & $-0.507 \pm 0.014$ & -0.507 & -0.0 & -0.507 & -0.0 \\
$\left(g_{L}^{\text {eff }}\right)^{2}$ & $0.3001 \pm 0.0014$ & 0.3042 & -2.9 & 0.3032 & -2.2 \\
$\left(g_{R}^{\text {eff }}\right)^{2}$ & $0.0308 \pm 0.0011$ & 0.0301 & +0.6 & 0.0299 & +0.8 \\
$Q_{W}(\mathrm{Cs})$ & $-72.18 \pm 0.46$ & -72.88 & +1.5 & -72.26 & +0.2 \\
$Q_{W}(\mathrm{Tl})$ & $-114.8 \pm 3.6$ & -116.7 & +0.5 & -115.7 & +0.3 \\
$\sum_{i=d, s, b}\left|V_{u i}\right|^{2}$ & $0.9917 \pm 0.0028$ & 1.0000 & -3.0 & 0.9902 & +0.5 \\
\hline
\end{tabular}

and performed a global fit to 22 observables. The best-fit values are

$$
\begin{aligned}
& \frac{v_{1}^{2}}{u^{2}}=0.00489, \quad \frac{v_{1}^{2}}{w^{2}}=0.00238 \\
& r=10.2, \quad y=0.0955, \quad x=0.135 .
\end{aligned}
$$

Our results are summarized in Table I.

We see that we are able to explain the apparent nonunitarity [1] of the quark mixing matrix and reduce the $\mathrm{NuTeV}$ discrepancy [3] while maintaining excellent agreement with precision data at the $Z$ resonance, except for the $b \bar{b}$ forwardbackward asymmetry measured at LEP, which is also not explained by the standard model. In fact, the shift of $A_{f b}^{0, b}$ is given in our model by

$$
\begin{aligned}
\Delta A_{f b}^{0, b} & =\frac{3}{4}\left(A_{e} \Delta A_{b}+A_{b} \Delta A_{e}\right) \\
& =-0.07 \Delta \sin ^{2} \theta_{q}-5.57 \Delta \sin ^{2} \theta_{l} .
\end{aligned}
$$

Because of the dominant coefficient of the second term, it measures essentially the same quantity as $A_{l}$ and there is no realistic means of reconciling the discrepancy of $\sin ^{2} \theta_{\text {eff }}$ at the $Z$ resonance using $b \bar{b}$ versus using leptons in the final state.

\section{Other Effects}

The new polarized $e^{-} e^{-} \rightarrow e^{-} e^{-}$experiment (E158) at SLAC (Stanford Linear Accelerator Center) is designed to measure the left-right asymmetry which is proportional to $G_{F}\left(1-4 \sin ^{2} \theta_{W}\right)$ to an accuracy of about $10 \%$. Using the standard-model prediction of $\sin ^{2} \theta_{W}=0.238$, our expectation is that the above measurement will shift by only $-2.2 \%$ from its standard-model prediction. The new polarized $e p$ elastic scattering experiment (Qweak) at TJNAF (Thomas Jefferson National Accelerator Facility) is designed to measure $Q_{W}$ of the proton to an accuracy of about $4 \%$. We expect a shift of only $+3.0 \%$. Using Eq. (37), we see also that the scale of new physics, i.e. $u$ and $w$, is at the $\mathrm{TeV}$ scale. Specifically, using the best-fit values of $r, y$, and $x$, we find $M_{W_{2}} \simeq M_{Z_{2}} \simeq 1.2 \mathrm{TeV}$, and $M_{Z_{3}} \simeq 0.8 \mathrm{TeV}$.

\section{Acknowledgments}

This work was supported in part by the China National Natural Science Foundation and the U. S. Department of Energy. The hospitality of XXIII ENFPC and its organizers (especially Maria Beatriz Gay Ducati) was greatly appreciated.

\section{References}

[1] H. Abele et al., Phys. Rev. Lett. 88, 211801 (2002).

[2] Particle Data Group, K. Hagiwara et al., Phys. Rev. D66, 010001 (2002).

[3] G. P. Zeller et al., NuTeV Collaboration, Phys. Rev. Lett. 88, 091802 (2002).

[4] M. Grunewald, Talk at ICHEP 2002 (Amsterdam).

[5] X. Li and E. Ma, hep-ph/0212029.

[6] X. Li and E. Ma, Phys. Rev. Lett. 47, 1788 (1981). 